Guide to

INVERTEBRATE ANIMALS 
Related titles

W. S. Bullough: Practical Invertebrate Anatomy, Second Edition

J. B. Jennings: Feeding, Digestion and Assimilation in Animals, Second Edition

A. J. Marshall and W. D. Williams: Textbook of Zoology: Invertebrates

(Seventh Edition of Parker and Haswell, Vol. I)

P. J. Mill: Respiration in the Invertebrates 


\title{
Guide to INVERTEBRATE ANIMALS
}

\author{
J. E. WEBB \\ Professor of Zoology, Westfield College, \\ University of London \\ J. A. WALLWORK \\ Reader in Zoology, Westfield College, \\ University of London \\ J. H. ELGOOD \\ Formerly Associate Professor of Zoology, \\ University of Ibadan, Nigeria
}

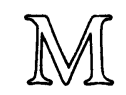


(c) J. E. Webb, J. A. Wallwork, J. H. Elgood 1975

Softcover reprint of the hardcover 1st edition 1975

All rights reserved. No part of this publication may be reproduced or transmitted, in any form or by any means, without permission

First published 1975 by

THE MACMILLAN PRESS LTD

London and Basingstoke

Associated companies in New York Dublin

Melbourne Johannesburg and Madras

SBN 333190068 (hard cover)

333190076 (paper cover)

ISBN 978-0-333-19007-4 ISBN 978-1-349-15599-6 (eBook)

DOI 10.1007/978-1-349-15599-6

This book is sold subject to the standard conditions of the Net Book Agreement.

The paperback edition of this book is sold subject to the condition that it shall not, by way of trade or otherwise, be lent, resold, hired out, or otherwise circulated without prior consent in any form of binding or cover other than that in which it is published and without a similar condition including this condition being imposed on the subsequent purchaser. 


\section{Preface}

This Guide to Invertebrate Animals has evolved over the past 35 years from lecture notes and cyclostyled information sheets given to university students in the United Kingdom and Africa. The original version, which was produced by two of us (JEW and JHE), was published in 1955 under the title Animal classification by the University of Ibadan Press, primarily for the use of students of that University. In the present volume, this Classification has been brought up to date and illustrated with numerous blackboard-style drawings to show the more important anatomical and morphological features of most of the animals concerned.

This book is intended for the use of sixth-formers and university undergraduates taking introductory courses in invertebrate biology, although its use may extend into fields of terrestrial, freshwater and marine ecology. By reducing to a pocket size format, the volume can be handled with equal ease in the field and the laboratory. The picture it attempts to present is one of an overa11, yet detailed, view of the diversity of invertebrate life, coupled with a scheme of classification which brings order to this diversity. Hopefully, the presentation of a considerable amount of basic information in this compact form will allow the reader to explore other aspects of zoology.

We would like to express our appreciation to various colleagues in Westfield College and outside who have given encouragement and made helpful suggestions during the preparation of this book. We owe a very special debt of gratitude to Mrs. Margaret Clarke, who prepared the typescript with accuracy, patience and willingness. 


\section{Contents}

Text Figure

1

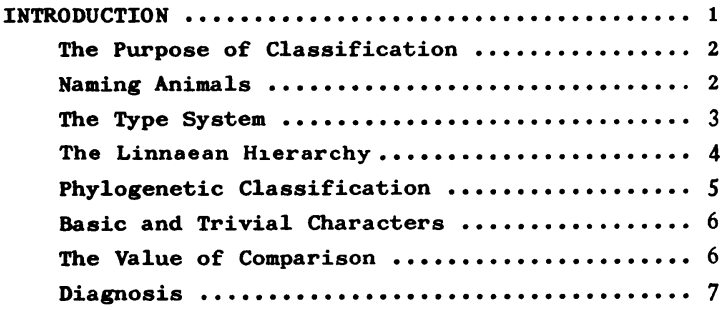

PROTISTA ............................. 9

Kingdom Animalia $\ldots \ldots \ldots \ldots \ldots \ldots \ldots \ldots \ldots . \ldots . \ldots 9$

Subkingdom \& Phylum Protista ............ 9

Class Mastigophora (Flagellata) ......... 10

Subclass Phytomastigophora .......... 11

Subclass Zoomastigophora ............ 11

Class Sarcodina $\ldots \ldots \ldots \ldots \ldots \ldots \ldots \ldots \ldots \ldots 11$

Subclass Rhizopoda ............... 11

Order Amoebida ................... 12

Order Foraminiferida ............. 12

Order Mycetozoida ................ 12

Subclass Actinopoda ............... 12

Order Radiolaria ............... 13

Order Heliozoa ................. 13

Class Sporozoa $\ldots \ldots \ldots \ldots \ldots \ldots \ldots \ldots \ldots \ldots \ldots$

Subclass Telosporea ............... 13

Order Coccidiomorpha ............. 14

Order Gregarinidea ............... 14

Subclass Piroplasmea ............... 14

Subclass Toxoplasmea ............... 14

Subclass Cnidospora ................. 14 
Class Ciliata ................. 15

Subclass Holotricha ............. 15

Subclass Spirotricha ............ 15

MESOZOA AND SPONGES .................. 20

Subkingdom \& Phylum Mesozoa ........... 20

Subkingdom Parazoa \& Phylum Porifera ...... 20

Class Calcarea ................... 21

Class Hexactinellida .............. 22

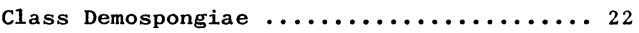

Subclass Tetractinellida ......... 22

Subclass Monaxonida ............. 22

Subclass Keratosa ............... 22

COELENTERATES AND CTENOPHORES $\ldots \ldots \ldots \ldots \ldots \ldots \ldots 24$

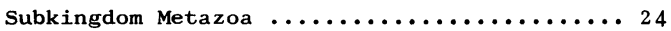

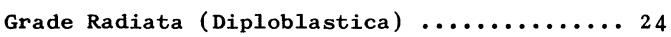

Phylum Coelenterata (Cnidaria) ......... 26

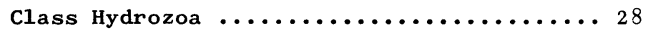

Order Hydroida ................. 29

Suborder Calyptoblastea .......... 29

Suborder Gymnoblastea .......... 29

Order Trachylina .............. 30

Suborder Trachymedusae ......... 30

Suborder Narcomedusae .......... 30

order Milleporina ............... 30

Order stylasterina ............. 31

Order Siphonophora ............. 31

Class Scyphozoa (Scyphomedusae) ........ 31

Order Lucernariida (Stauromedusae) .... 32

Order Charybdeida (Cubomedusae) ..... 33

Order Coronatae (Peromedusae) ...... 33

Order Semaeostomeae .............. 34

Order Rhizostomeae ................ 34

Class Anthozoa (Actinozoa) .............. 34

Subclass Alcyonaria ............. 35

Order Alcyonacea ................. 36

Order Stolonifera ................. 36

Order Pennatulacea ................ 36 


\begin{tabular}{|c|c|c|}
\hline & Text & Figure \\
\hline Order & Gorgonacea $\ldots \ldots \ldots \ldots \ldots \ldots \ldots$ & 45 \\
\hline \multicolumn{3}{|c|}{ Subclass Zoantharia .............. 37} \\
\hline Order & Actinaria $\ldots \ldots \ldots \ldots \ldots \ldots \ldots$ & 44 \\
\hline Order & Antipatharia $\ldots \ldots \ldots \ldots \ldots \ldots 37$ & 45 \\
\hline Order & Madreporaria $\ldots \ldots \ldots \ldots \ldots \ldots \ldots$ & 45 \\
\hline Order & Ceriantharia $\ldots \ldots \ldots \ldots \ldots \ldots$ & 38 \\
\hline \multicolumn{3}{|c|}{ lum Ctenophora .................. 46} \\
\hline \multicolumn{3}{|c|}{ lass Tentaculata $\ldots \ldots \ldots \ldots \ldots \ldots \ldots \ldots \ldots \ldots 47$} \\
\hline Order & Cydippida $\ldots \ldots \ldots \ldots \ldots \ldots \ldots \ldots 47$ & 48,49 \\
\hline Order & Cestida $\ldots \ldots \ldots \ldots \ldots \ldots \ldots \ldots 4$ & 50 \\
\hline Order & Lobata $\ldots \ldots \ldots \ldots \ldots \ldots \ldots \ldots$ & 50 \\
\hline Order & Platyctenea $\ldots \ldots \ldots \ldots \ldots \ldots \ldots 4$ & 50 \\
\hline Nuda & & \\
\hline Order & Beroida $\ldots \ldots \ldots \ldots \ldots \ldots \ldots \ldots$ & 50 \\
\hline
\end{tabular}

ACOELOMATE ANIMALS $\ldots \ldots \ldots \ldots \ldots \ldots \ldots \ldots \ldots \ldots \ldots \ldots \ldots$

Grade Bilateria (Triploblastica) ........ 51

Subgrade Acoelomata ................ 51

Phylum Platyhelminthes .............. 52

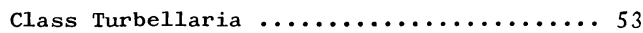

Order Acoela .................. 54

Order Rhabdocoela .............. 54

Order Tricladida ............. 54

order Polycladida .............. 54

Class Temnocephaloidea ............. 56

Class Monogenea ................ 56

Subclass Monopisthocotylea ......... 56

Subclass Polyopisthocotylea ........ 57

Class Aspidogastrea .............. 57

Class Digenea ................... 57

Class Didymozoidea ................ 60

Class Cestodaria ..................60

Class Cestoda ................... 60

Subclass Didesmida ..............6 61

Subclass Tetradesmida .............6 61

Phylum Nemertea $\ldots \ldots \ldots \ldots \ldots \ldots \ldots \ldots \ldots . \ldots 6$

Class Anopla .................... 64

Order Palaeonemertini ...........6 64

Order Heteronemertini ............ 64

vii 
Class Enopla ..................... 64

Order Hoplonemertini .............6 64

Order Bdellonemertini ............ 64

PSEUdOCOELOMATE ANIMALS ................ 67

Subgrade Pseudocoelomata ............. 67

Phylum Nematoda ................... 68

Class Aphasmidia ................... 69

Class Phasmidia .................. 69

Phylum Nematomorpha ............... 72

Phylum Kinorhyncha ................ 73

Phylum Rotifera .................. 74

Phylum Gastrotricha ................. 75

Phylum Endoprocta ................. 76

Phylum Acanthocephala .............. 77

7 EUCOELOMATE ANIMALS: ANNELIDA ............. 79

Subgrade Eucoelomata ................ 79

Phylum Annelida ................... 81

Class Polychaeta ................. 84

Order Errantia ................. 84

Order Sedentaria ................ 85

Class 01igochaeta ................. 85

Order Plesiopora ................ 85

Order Prosotheca ................ 86

Order Prosopora ................. 86

Order Opisthopora ............... 86

Class Archiannelida .............. 87

Class Hirudinea .................. 87

Order Acanthobdellida ............ 87

Order Rhynchobdellida ............. 88

Order Gnathobdellida ............. 88

Order Erpobdellida ............... 88

8 ARTHROPODS: TRILOBITES \& CHELICERATES ........ 92

Arthropods ...................... 92

Phylum Trilobitomorpha $\ldots \ldots \ldots \ldots \ldots \ldots \ldots . \ldots 9$

Class Trilobita $\ldots \ldots \ldots \ldots \ldots \ldots \ldots \ldots$ 
Phylum Chelicerata ................. 97

Class Merostomata ................ 97

Order Eurypterida ............... 98

Order Xiphosura .................. 98

Class Arachnida .................... 99

Order Scorpiones ...................100

Order Chelonethi ................. 100

Order solifugae .................. 101

Order Palpigradi .................. 101

order Schizopeltida ............... 102

Order Holopeltida ................ 102

Order Amblypygi ................... 103

Order Araneae .................... 103

Order Ricinulei .................. 104

Order opiliones .................. 104

106

Order Acari .................... 105

Class Pycnogonida ................. 105

BIRAMOUS ARTHROPODS: CRUSTACEANS ......... 108

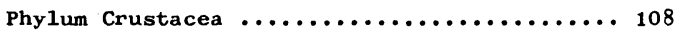

Class Cephalocarida ................ 110

Class Branchiopoda .................. 110

Order Anostraca .................. 110

128

Order Notostraca ................. 111

Order Diplostraca ................. 111

Suborder Conchostraca ............ 111

Suborder cladocera .............. 112

Class 0stracoda ................... 112

Class Mystacocarida ................ 113

Class Copepoda .................... 113

Class Branchiura .................. 114

Class Cirripedia ................. 114

Order Thoracica ................ 115

Suborder Pedunculata ............. 115

Suborder operculata ............. 115

Order Acrothoracica ............... 115

Order Ascothoracica ............... 116

Order Rhizocephala ................ 116 


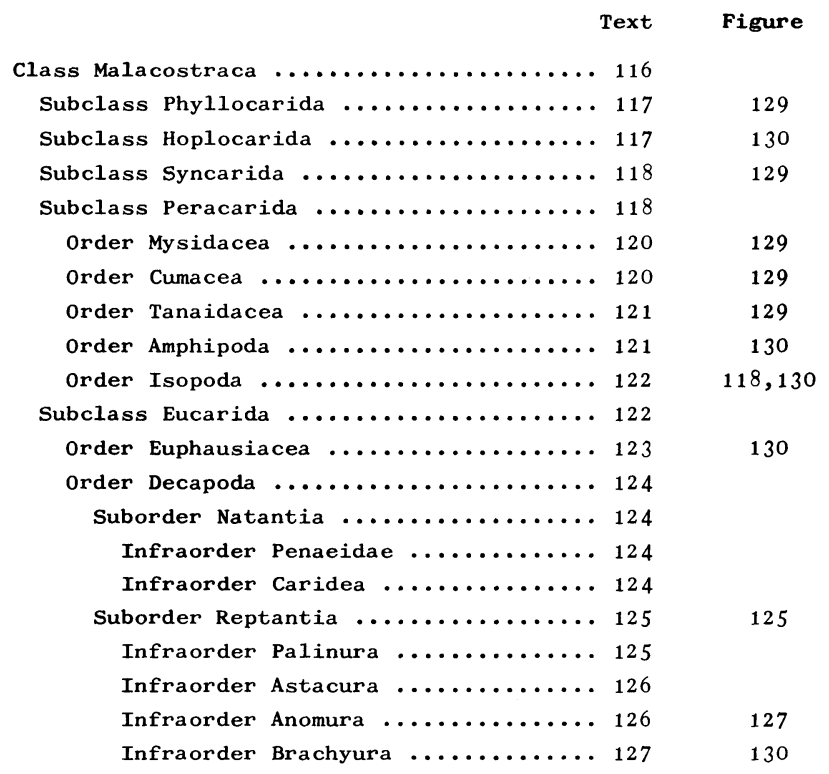

10 UNIRAMOUS ARTHROPODS: ONYCHOPHORA, MYRIAPODA,

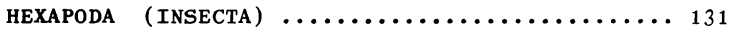

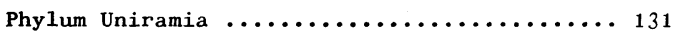

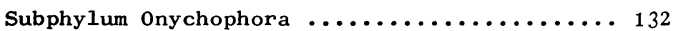

Subphylum Myriapoda ............... 132

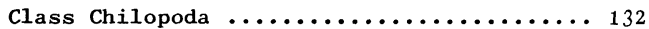

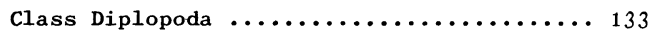

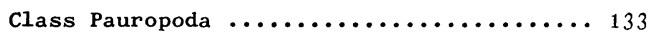

Class Symphyla .................. 133

Subphylum Hexapoda ................ 136

Class Collembola .................. 137

Class Protura ..................... 137

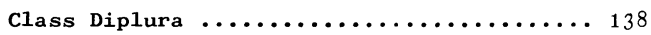

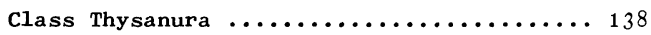

Class Pterygota ................... 141

Infraclass Palaeoptera ............ 141

Order Plectoptera .............. 142 


\begin{tabular}{|c|c|c|}
\hline & Text & Figure \\
\hline order & odonata $\ldots \ldots \ldots \ldots \ldots \ldots \ldots \ldots \ldots \ldots 142$ & 143 \\
\hline \multicolumn{3}{|c|}{ Infraclass Polyneoptera $\ldots \ldots \ldots \ldots \ldots \ldots \ldots$} \\
\hline Order & Dictyoptera ................ 145 & \\
\hline Order & Isoptera .................. 145 & \\
\hline Order & Zoraptera .................. 146 & \\
\hline Order & Plecoptera .................... 146 & 150 \\
\hline Order & Notoptera ................... 147 & \\
\hline Order & Cheleutoptera .............. 147 & \\
\hline Order & Orthoptera ................ 148 & 150 \\
\hline Order & Embioptera $\ldots \ldots \ldots \ldots \ldots \ldots \ldots \ldots \ldots$ & \\
\hline Order & Dermaptera .................. 149 & 149 \\
\hline \multicolumn{3}{|c|}{ Infraclass Paraneoptera .............. 151} \\
\hline Order & Psocoptera ................ 152 & 156 \\
\hline Order & Mallophaga ................. 152 & \\
\hline Order & Anoplura .................. 153 & \\
\hline Order & Thysanoptera $\ldots \ldots \ldots \ldots \ldots \ldots \ldots 153$ & 156 \\
\hline Order & Homoptera .................. 154 & 154,156 \\
\hline Order & Heteroptera .............. 155 & 155,156 \\
\hline \multicolumn{3}{|c|}{ Infraclass 0ligoneoptera $\ldots \ldots \ldots \ldots \ldots \ldots 157$} \\
\hline Order & Coleoptera $\ldots \ldots \ldots \ldots \ldots \ldots \ldots \ldots 158$ & 164 \\
\hline Order & Megaloptera ................. 158 & \\
\hline Order & Raphidioptera .............. 159 & \\
\hline Order & Planipennia $\ldots \ldots \ldots \ldots \ldots \ldots \ldots \ldots$ & \\
\hline Order & Mecoptera .................. 160 & \\
\hline Order & Trichoptera ................ 160 & \\
\hline Order & Lepidoptera $\ldots \ldots \ldots \ldots \ldots \ldots \ldots \ldots 161$ & 164 \\
\hline Order & Diptera $\ldots \ldots \ldots \ldots \ldots \ldots \ldots \ldots \ldots 161$ & 164 \\
\hline Order & Siphonaptera $\ldots \ldots \ldots \ldots \ldots \ldots \ldots \ldots 162$ & \\
\hline Order & Hymenoptera $\ldots \ldots \ldots \ldots \ldots \ldots \ldots \ldots 162$ & 164 \\
\hline Order & Strepsiptera ................. 163 & 163 \\
\hline
\end{tabular}

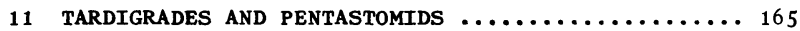

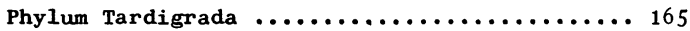

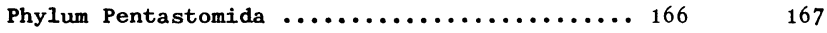

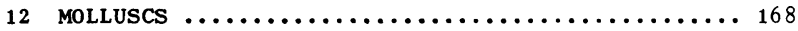

Phylum Mollusca .................. 168

Class Monoplacophora .................. 170 


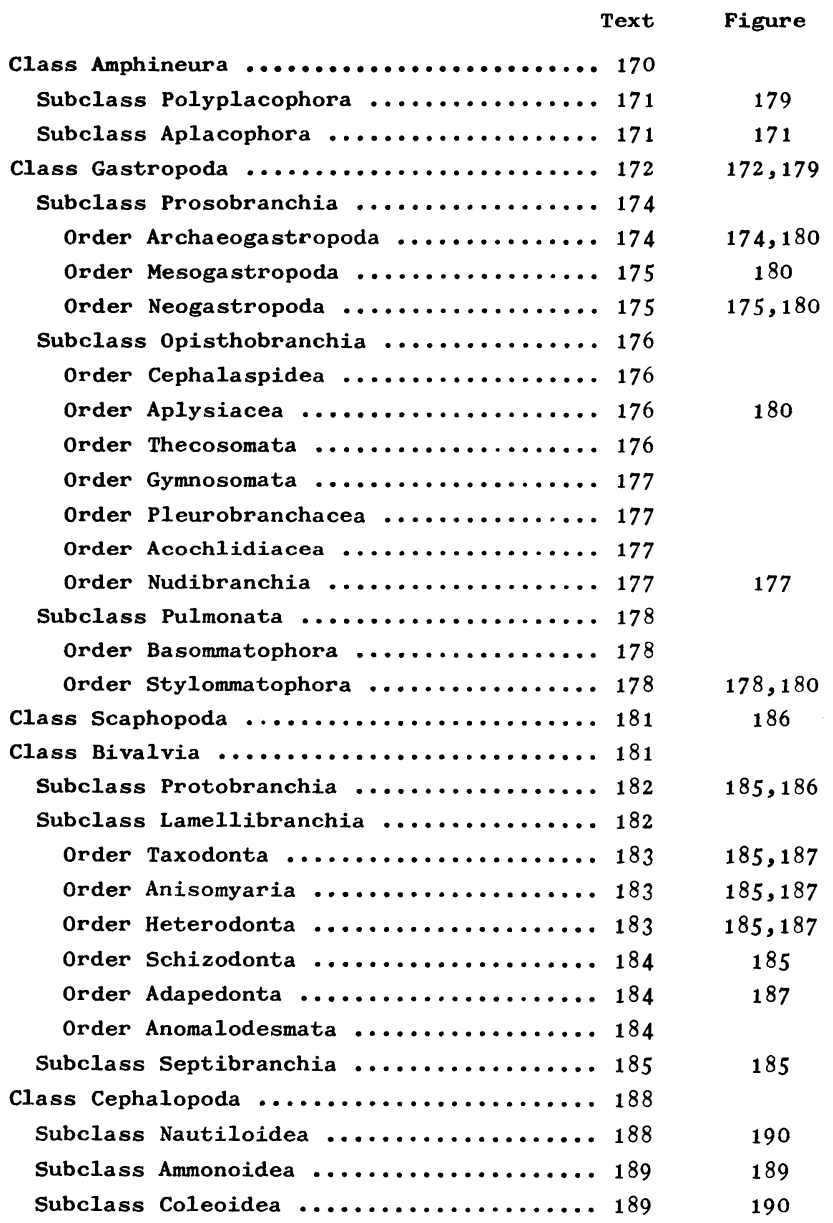

13 MINOR EUCOELOMATE PHYLA ................. 191

Phylum Echiurida ....................... 193

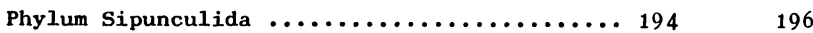

Phylum Priapulida ................... $195 \quad 196$ 


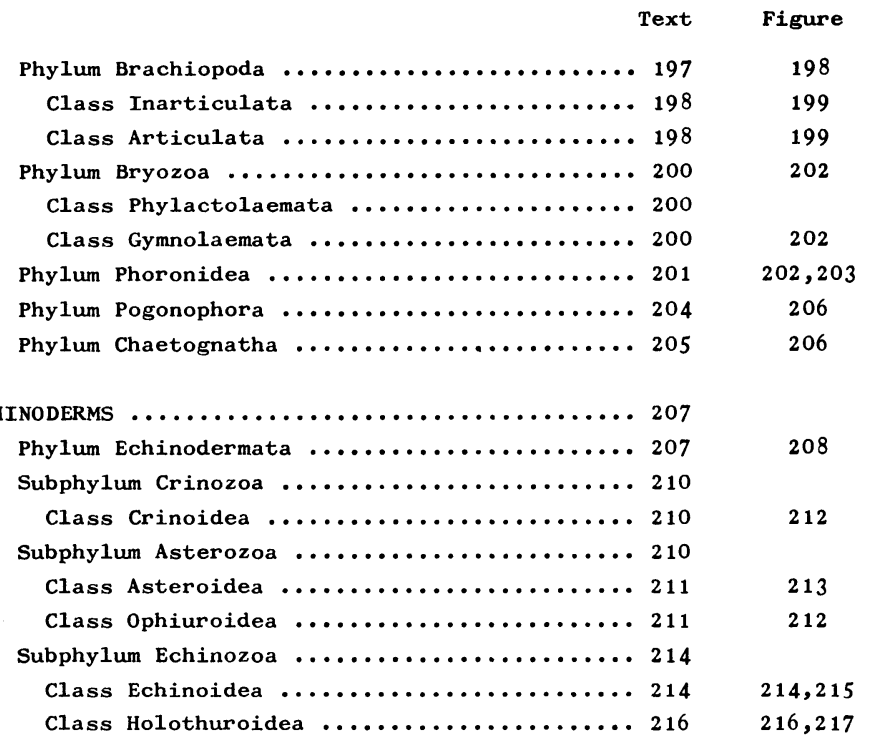

15 HEMICHORDATES AND CHORDATES .............. 218

Phylum Hemichordata ............... 219

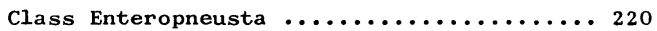

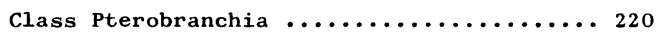

220,221

221

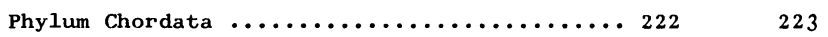

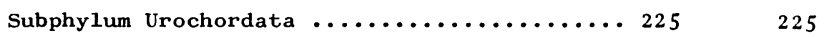

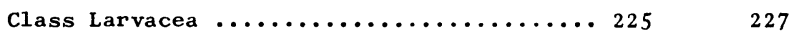

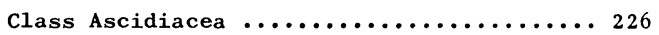

Order Enterogona ...............226 227

Order Pleurogona ................226 226

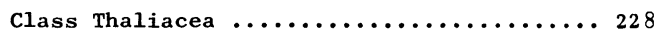

Order Pyrosomida ................ 228

Order Salpida .................. 229

228,230

230

Order Doliolida ................. 229

Subphylum Cephalochordata ............231

231,232

Subphylum Craniata ................. 233

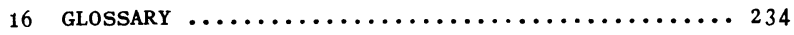

